\title{
Optimizing Preparation of Graphene Oxide Grids for Cryo-EM
}

Giovanna Grandinetti ${ }^{1}$ and Yoshie Narui ${ }^{2}$

${ }^{1}$ The Ohio State University, CEMAS, United States, ${ }^{2}$ The Ohio State University, CEMAS, Columbus, Ohio, United States

The use of graphene oxide (GO) has been shown to improve sample dispersion for single particle cryoEM data collection. There are many different published methods on how to fabricate GO grids, with each method having its own pros and cons. Our aim was to test as many of these methods as possible using the same equipment and starting materials and to report which methods were able to provide the most consistent results in our hands. Here, we present data on how different factors affect GO distribution across Quantifoil grids. For these experiments, commercially available graphene oxide solution from SigmaAldrich was used. We have tested the following effects on GO sheet dispersion: (1) different glow discharge currents and times, (2) different methods of depositing the GO sheets on to the grids, and (3) different additives. The goal is to consistently achieve single layer GO coverage over the majority of holes on a Quantifoil TEM grid.

We sought to initially optimize the drop-cast method of GO sheet deposition due to its relative ease and quick set-up time. Drop-cast experiments were performed using the methods described by Pantelic et al. [1] and Martin et al. [2] using different glow discharge parameters for Quantifoil grids (Figure 1). In our experience, using $20 \mathrm{~mA}$ for 150 or 120 s provided adequate coverage but most holes in the Quantifoil TEM grid had multiple layers of GO or wrinkled GO sheets. In order to obtain more uniform GO sheet coverage, we have tried using octyl-D-glucoside (OG) detergent. Non-ionic detergent has been used in the past to prepare graphene oxide grids, and OG detergent is compatible with many of our biological samples. We still observed wrinkles and holes with multiple layers of GO (Figure 2 b, c). Next, we tried combining drop-cast methods with methods described by Palovcak et al. [3]. In this method, GO solution was diluted in a 1:5 water:methanol mixture. The GO solution was then sonicated and centrifuged to pellet out larger GO sheets. The pellet was resuspended in 1:5 water:methanol, and added to Quantifoil grids using the drop-cast method (Figure $2 \mathrm{~d}$, e). Additional additives were tested to maximize the most holes with single layer coverage. It was found that sonication and centrifugation helped isolate larger GO sheets, and that adding detergents helped smooth the sheets over the holes. Using the drop-cast technique resulted in a gradient of GO coverage across TEM grids, however the grids were more robust and could tolerate more movement after blotting away the GO solution. Using methods that float GO sheets on to TEM grids resulted in more uniform coverage, but the grids were more fragile and required drying slowly overnight to obtain sufficient GO coverage.

The authors acknowledge Daniel Veghte and Henk Colijn for their many useful discussions and contributions to this work. 


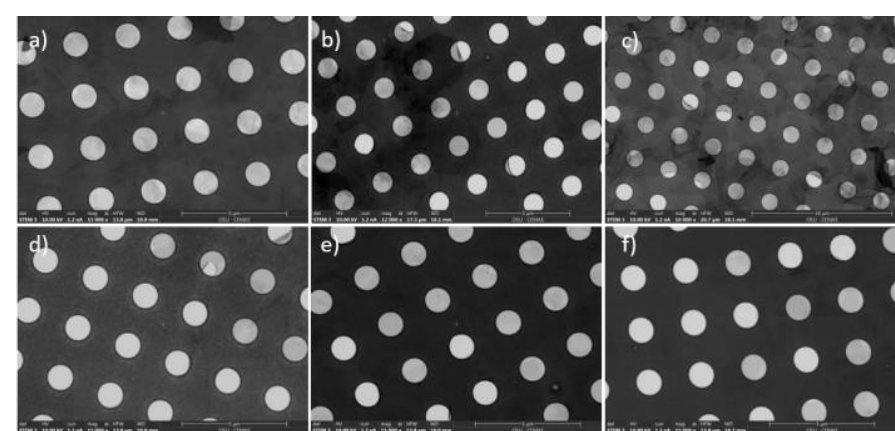

Figure 1. Different currents and times were tested to optimize glow discharge parameters for GO grids using a Pelco EasiGlow system: a) $30 \mathrm{~mA}$ for $150 \mathrm{sec}$, b) $30 \mathrm{~mA}$ for $120 \mathrm{sec}$, c) $30 \mathrm{~mA}$ for $90 \mathrm{sec}$, d) 20 $\mathrm{mA}$ for $150 \mathrm{sec}$, e) $20 \mathrm{~mA}$ for $120 \mathrm{sec}$, and f) $20 \mathrm{~mA}$ for $90 \mathrm{sec}$. Using $20 \mathrm{~mA}$ for either 150 or $120 \mathrm{sec}$ resulted in the most holes with a single layer of GO, but most holes were still either uncovered or had multiple GO sheets over them, which is not optimal for automated high-resolution data collection

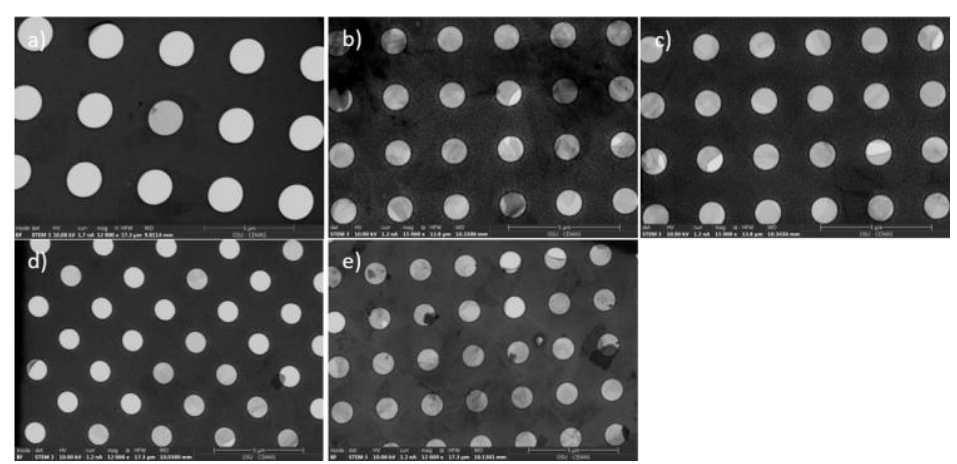

Figure 2. Different concentrations and different additives were chosen to observe the effects on graphene oxide (GO) sheet deposition: a) GO sheets in water, $0.02 \mathrm{mg} / \mathrm{mL}, 3 \mu \mathrm{L}$ added to grid, b) GO sheets in water, $0.01 \mathrm{mg} / \mathrm{mL}, 0.003 \%$ v/v OG, $5 \mu \mathrm{L}$ added to grid, c) GO sheets in water, $0.01 \mathrm{mg} / \mathrm{mL}, 0.003 \% \mathrm{v} / \mathrm{v}$ OG, $3 \mu \mathrm{L}$ added to grid, d) GO sheets in 1:5 water:methanol, $0.2 \mathrm{mg} / \mathrm{mL}, 3 \mu \mathrm{L}$ added to grid, and e) GO sheets in 1:5 water:methanol, $0.05 \mathrm{mg} / \mathrm{mL}, 3 \mu \mathrm{L}$ added to grid. Grids were imaged using a ThermoFisher Quattro SEM with STEM detector in brightfield mode.

\section{References}

[1] R.S. Pantelic, J.C. Meyer, U. Kaiser, W. Baumeister, and J.M. Plitzko. Journal of Structural Biology 170 (2010), p. 152-156.

[2] T.G. Martin, A. Boland, A.W.P. Fitzpatrick, and S.H.W. Scheres. Figshare (2016): Graphene Oxide Grid Preparation. figshare. Media. https://doi.org/10.6084/m9.figshare.3178669.v1.

[3] E. Palovcak, F. Wang, S.Q. Zheng, Z. Yu, S. Li, M. Betegon, D. Bulkley, D.A. Agard, and Y. Cheng. Journal of Structural Biology 204 (2018), p. 80-84. 